\title{
Fiber Optic Detection of Ammonia Gas
}

\author{
L. Kalvoda, J. Aubrecht, R. Klepáček
}

\begin{abstract}
Bathochromic shifts accompanying the formation of several bivalent metallic complexes containing 5-(4'-dimethylaminophenylimino) quinolin-8-one (L1), and 7-chlore-5(4'-diethylamino-2-methylphenylimino) quinolin-8-one (L2) ligands in ethanol solutions were evaluated by VIS-NIR spectroscopy. The [L1-Cu-L1] sulphide complex was selected as a reagent for further tests on optical fibres. Samples of multimode siloxane-clad fused-silica fibre were sensitized by diffusing an ethanol/chloroform solution of the dye into the cladding polymer, and tested by VIS-NIR optical spectroscopy (12 cm long fibre sections), and optical time domain reflectometry (OTDR; 20 ns laser pulses, wavelength $850 \mathrm{~nm}, 120 \mathrm{~m}$ long fibre sensitized within the interval 104-110 m). A well-resolved absorption band of the reagent could be identified in the absorption spectra of the fibres. After exposure to dry ammonia/nitrogen gas with increasing ammonia concentration $(0-4000$ ppm), the short fibre samples showed subsequent decay of NIR optical absorption; saturation was observed for higher ammonia levels. The concentration resolution $r \approx 50 \mathrm{ppm}$ and forward response time $\tau_{90} \approx 30 \mathrm{sec}$ were obtained within the interval $0-1000 \mathrm{ppm}$. The OTDR courses showed an enhancement of the back-scattered light intensity coming from the sensitized region after diffusion of the initial reagent, and decay after exposure to concentrated ammonia/nitrogen gas (10000 ppm).
\end{abstract}

Keywords: chemical sensors, ammonia sensors, optical fibres.

\section{Introduction}

Rapid detection and location of ammonia gas leaks poses a serious problem at all facilities utilising almost pure ammonia gas, such as large-scale refrigerating systems (breweries, dairies, abattoirs, logistic centres, ice-rinks, etc.), chemical plants producing ammonia by the Haber-Bosh reaction, and fertilizer plants. Every year, several accidents caused by ammonia are reported, resulting in severe damage to health and general distress. The concentration limit of human ammonia perception is about $50 \mathrm{ppm}$, but even lower concentrations are harmful for the human respiratory system. The long-term allowed concentration is defined as $20 \mathrm{ppm}$. Higher concentrations (500-1000 ppm) lead to a serious attack on the respiratory system. The lethal concentration limit is estimated as 5000-10000 ppm [1]. Such a level can be readily reached in the event of a highly concentrated ammonia gas leak.

The standard architecture of an ammonia detection system used at present is based on a network of individual conductometric sensing heads equipped with a metal-oxide film [1]. Clearly, both the cost and the electric power consumption of such a sensing network rise rapidly with the number of sensors installed. Low gas selectivity and insufficient long-term stability of the sensing elements are also serious problems [1].

Numerous sensing schemes and optical systems have been tested to overcome these drawbacks (e.g. [1, 3-8]). One approach employs a suitably sensitized optical fibre. The two most extensively tested principles of this type are based on absorption- and fluorescence-based intrinsic sensing fibres. Selected reagent is embedded in the fibre cladding; ammonia gas then induces spectral variations of the fibre optical absorbance or fluorescence through its chemical reaction with the reagent. Short sections of such a fibre can be employed in fabricating the individual sensing heads, whereas various modifications of the optical reflectometry method (optical time domain reflectometry, OTDR, optical frequency domain reflectometry, OFDR, optical low coherence reflectometry, OLCR, optical time-of-flight chemical detection, OTOF-CD) provide a basis for constructing distributed sensing systems [9-11].

The crucial part of any intrinsic fibre optic sensor design is the selection of a reaction mechanism transforming an analyte exposure event into variations of the optical properties of the fibre. For instance, a pH-sensitive dye can be embedded into the fibre cladding. When exposed to alkaline ammonia gas, the optical absorption band of the dye is shifted; the change in cladding absorption modifies the light spectrum (guided in the fibre core as individual waveguide modes) through evanescent electromagnetic field components. Unfortunately, such utilization of an acid base reaction suffers from its strong dependence on the humidity level (the presence of hydronium ions is necessary for the creation of $\mathrm{NH}_{4}^{+}$ions) and the obvious low sensing selectivity. The latter drawback can be partly compensated by using a proper gas selective membrane [1].

The ammonia detection approach employed here uses the ligand exchange reaction proposed in [12]. It is in principle capable reducing the humidity dependence and enhancing the selectivity in comparison with the acid base reaction (see the Experimental section for more details). In the research stage described here, the following objectives were adopted: (i) to choose reagent(s) suitable for further studies on optical fibres by evaluating the optical absorption spectra of two suitable organic dyes and their metallic complexes in solution, (ii) to prepare sensing fibre samples by diffusion of the reagent into a plastic clad silica (PCS) fibre cladding using the frequently applied procedure elaborated by our research group in frame of the CEC Copernicus programme [13, 9, 14], (iii) to test the concentration and temporal response to ammonia gas exposure by VIS-NIR absorption spectroscopy measurements on short fibre sections, and (iv) to pre-evaluate the qualitative features of an OTDR signal by measurements on a longer fibre, only partially sensitized with the selected reagent. Preparation, optimization, and quantitative characterization of a real distributed fibre optic ammonia sensor will be carried out in the next step, and the results will be published in a forthcoming paper. 


\section{Experimental}

\subsection{Chemicals}

Ethanol, chloroform, and acetone (p.a. grade) were obtained from Lach-Ner, CR; deionised water $\left(0.1 \mu \mathrm{S} \mathrm{cm}^{-1}\right)$ was employed. 5-(4'-dimethylaminophenylimino) quinolin-8-one $(\mathrm{L} 1, \mathrm{Mw}=277.38)$, and 7-chlore-5(4- diethylamino-2-methylphenylimino) quinolin-8-one $(\mathrm{L} 2, \mathrm{Mw}=353.85)$ was synthesized as described in [13]. $\mathrm{FeBr}_{2}, \mathrm{CoBr}_{2}, \mathrm{NiCl}_{2}, \mathrm{CuCl}_{2}$, $\mathrm{CuSO}_{4}, \mathrm{ZnSO}_{4}, \mathrm{Cd}\left(\mathrm{NO}_{3}\right)_{2}, \mathrm{Hg}\left(\mathrm{NO}_{3}\right)_{2}, \mathrm{~Pb}\left(\mathrm{NO}_{3}\right)_{2}$, and $\mathrm{NaOH}$ were obtained from Sigma-Aldrich and Lach-Ner. Solutions of metallic complexes were prepared by admixing $50 \mathrm{ml}$ of $\mathrm{L} 1$ or $\mathrm{L} 2$ solution $(0.01 \mathrm{M})$ in ethanol and $2.5 \mathrm{ml}$ of the metallic salt solution in an ethanol/water mixture $(0.1 \mathrm{M})$ and stirring for 30 min at RT. The amount of water was kept at a minimal level. The complex dye formation was then checked by VIS-NIR absorption spectroscopy. Fresh solutions of the complex dyes were used in subsequent preparation of the sensing fibres. The following complexes were prepared and tested: [L-Fe-L] $\mathrm{Br}_{2}$, [L-Co-L] $\mathrm{Br}_{2}$, [L-Ni-L] Cl${ }_{2}$, [L-Cu-L] $\mathrm{Cl}_{2}$, [L-Zn-L] $\mathrm{SO}_{4},[\mathrm{~L}-\mathrm{Cd}-\mathrm{L}]\left(\mathrm{NO}_{3}\right)_{2},[\mathrm{~L}-\mathrm{Hg}-\mathrm{L}]\left(\mathrm{NO}_{3}\right)_{2},[\mathrm{~L}-\mathrm{Pb}-\mathrm{L}]$ $\left(\mathrm{NO}_{3}\right)_{2}$, (L stands for L1 or L2). Nitrogen gas (99.996 \% wt.) was obtained from Messer Technogas, CR. A dry ammonia/nitrogen mixture (10000 $\mathrm{ppm} \mathrm{NH}_{3}$ ) was prepared by evaporating a known amount of ammonia gas from ammonia liquor, drying it in a column filled with $\mathrm{NaOH}$, and mixing it with nitrogen gas.

\subsection{Preparation of tested fibres}

A custom-made PCS fibre $\left(n_{c}=1.458,200 / 260 \mu \mathrm{m}\right.$ core/cladding diameter, Dow Corning Optigard siloxane cladding, produced at IRE ASCR, Prague, CR) was utilized throughout the experiments. The fibre sections were washed in acetone for at least $12 \mathrm{~h}$ prior to further sensitization. The reagent was soaked into the fibre cladding from its etha$\mathrm{nol} /$ chloroform solution (1:1 wt.), washed in ethanol, and left to dry for $12 \mathrm{~h}$ in room conditions. Two types of fibres were prepared: short sections $(12 \mathrm{~cm})$ intended for VIS-NIR absorption spectroscopy measurements and a long fibre $(120 \mathrm{~m}$, sensitized within the interval 104-110 m) tested by OTDR. The whole preparation and subsequent characterization of the fibres was performed at room temperature (RT).

\subsection{Ligand exchange reaction}

The ammonia sensing fibres prepared in this work employ the ligand exchange reaction

$$
\begin{aligned}
{[L-\mathrm{Me}-\mathrm{L}]^{2+} } & +A^{2-}+n \mathrm{NH}_{3} \rightleftharpoons \\
& \rightleftharpoons\left[\left(\mathrm{NH}_{3}\right)_{n}-\mathrm{Me}\right]^{2+}+A^{2-}+2 L
\end{aligned}
$$

where $L$ is an organic chromatic ligand, $M e$ is a bivalent positive metal ion forming a complex ion with $L$ (see also Table 1), $A$ is a suitable counter-anion, and $n$ is the integer number, which depends on the type of $M e$ co-ordination. The superscripts indicate the degree of ionization of the particular fragment.

\subsection{Experimental characterization}

The VIS-NIR absorption spectra of the solutions and short fibre sections were measured using a setup containing a white light source (halogen lamp, $25 \mathrm{~W}$ ), a cuvette holder with focusing optics (solution spectroscopy measurements) or a sealed testing chamber attached to a gas mixing system (spectroscopy measurements of fibre sections), an optical pigtail collecting light from the sample under test and transferring it into an Ocean Optics S1000 array spectrometer (200-1200 $\mathrm{nm}$ wavelength range, $1 \mathrm{~nm}$ resolution, single channel operation) controlled by a PC. In the case of fibre testing, the light beam was focused into the tested fibre through a microscopic objective and collected by an integration sphere ( $1 \mathrm{~mm}$ in diameter) at the end of the pigtail. The light source, the testing chamber, and the collecting pigtail were all placed on a common optical rail.

The OTDR setup consisted of a Photodyne 5500 XFA OTDR unit (laser diode wavelength $\lambda=850 \mathrm{~nm}$, pulse width $20 \mathrm{~ns}$, average pulse power $30 \mu \mathrm{W}$, repeating frequency $3.1 \mathrm{kHz}$ ), HP 54615B digital oscilloscope (maximum sampling speed $1 \mathrm{GSa} / \mathrm{s}$, signal bandwidth $500 \mathrm{MHz}$ ), a sealed testing chamber $\left(4 \mathrm{dm}^{3}\right)$ attached to the cylinder with 10000 ppm ammonia/nitrogen gas, and a PC controller. The OTDR unit launched laser pulses into the tested fibre, registered a back-scattered light intensity using an internal PIN Si diode, and logarithmically amplified the detected signal. The temporal signal course was then recorded by the digital oscilloscope and transferred to the PC. The final pulse width limited the spatial resolution along the fibre length to $\sim 4 \mathrm{~m}$ (cf Eq. 3). The OTDR curves showing a full temporal course were recorded with the oscilloscope set to collect data within the time range $\Delta t=2000 \mathrm{~ns}$. The curve details were examined with $\Delta t=200 \mathrm{~ns}$. A numeric data averaging procedure (128 single-shot spectra were accumulated) was employed to reduce the signal-to-noise ratio of the resulting OTDR curves.

Variable concentrations of ammonia gas in nitrogen were prepared by volumetrically dosing the concentrated ammonia/nitrogen mixture (10000 ppm) into the gas circuit (including the measuring chamber) filled with nitrogen. A membrane circulation pump included in the circuit provided fast homogenization of the prepared gas mixture.

\subsection{Basic theory}

The VIS-NIR absorption spectra were obtained by a standard absorption spectroscopy method [15].

The OTDR technique relies on interrogation of an attached optical fibre by short monochromatic laser pulses, and a subsequent temporal analysis of the light intensity $P(x)$ returning to the OTDR unit from the fibre distance $x$, $x=\Delta t c / n_{c}\left(c-\right.$ light velocity in a vacuum, $n_{c}-$ refractive index of the fibre core, $\Delta t-$ time interval between the pulse onset and the measuring time point). Two types of processes have to be considered as the dominant signal sources in the case of an intrinsic absorption-based sensing fibre: Rayleigh scattering along the fibre length, and Fresnel reflection from the fibre splices and the free end [16].

The recorded OTDR curves $(\log (P(x)$ versus $x)$ provided information about the immediate optical properties of the tested fibre along its length. The total Fresnel reflection intensity $P^{F}(x)$ is proportional to the forward pulse power $P^{+}(x)$ at position $x$, multiplied by the square of the corresponding reflection coefficients $R_{i}$ averaged over all reflected light modes $M$ guided within the multimode fibre of the core diameter $D$ and numeric aperture $N A[15,17]$ 


$$
\begin{aligned}
& P^{F}(x) \approx P^{+}(x) \frac{1}{M} \sum_{i=1}^{M} R_{i}(x)^{2} ; \\
& M \approx 2 \pi^{2}\left(\frac{D}{\lambda}\right)^{2} N A^{2} ; \\
& P^{+}(x) \approx \int_{0}^{w} I_{L} \exp \{-a(x)\} \mathrm{d} x .
\end{aligned}
$$

The integration runs over pulse spatial width $w=\tau c / n_{c} ; \tau$ is the pulse duration. The intensity of the Rayleigh back-scattered light can be expressed as [10, 16, 18]

$P^{R}(x)=\frac{C}{2 n_{c}^{2}} \int_{0}^{w} I_{L} S_{B}(x) S_{R}(x) N A^{2}(x) \exp \{-2 a(x)\} \mathrm{d} x$,

where $C$ is a constant characterizing the coupling efficiency of the optics, $I_{L}$ is the laser intensity injected into the fibre, $S_{B}$ and $S_{R}$ are respectively a back-scattering factor and the Rayleigh scattering coefficient at position $x$. The latter relates on a microscopic level to the local polarizability $\alpha(x)$ of the fibre as [15]

$$
S_{R}(x) \approx \frac{\alpha(x)^{2}}{\lambda^{4}} .
$$

The total fibre attenuation $a(x)$ caused by scattering and absorption effects is obtained as [9]

$$
a(x)=\int_{0}^{x} a^{\prime}(l) \mathrm{d} l
$$

where $a^{\prime}(l)$ is a local attenuation coefficient and the integration runs over the fibre length $0-x$.

For example, in the case of a uniform fibre $\left(S_{R}(x), S_{B}(x)\right.$, $N A(x)$, and $a(x)$ independent of $x)$, it follows from (3) that the Rayleigh contribution to the OTDR logarithmic output, $\log \left(P^{R}(x)\right)$, should be a linear function of $x$. Contingent variations of $a^{\prime}(x)$ and/or $S_{R}(x), S_{B}(x)$ and $N A(x)$ along the fibre length lead respectively to the corresponding slope changes and/or local extremes appearing on the resulting OTDR curve.

\section{Results and discussion}

As expected, dyes L1 and L2 showed strong absorption bands in the spectral range $500 \mathrm{~nm}-800 \mathrm{~nm}$ with maxima at $626 \mathrm{~nm}$ and $690 \mathrm{~nm}$, respectively (Fig. 1). Creation of metallic complexes of the dyes resulted in bathochromic shifts $(\Delta \lambda)$ of the absorption bands due to widening of the corresponding electronic resonant systems. The complex ions containing ligand L1 showed remarkably larger shifts than those containing ligand L2; the greatest $\Delta \lambda$-value was observed for complex dye [L1-Cu-L1] $\mathrm{SO}_{4}$ (Table 1, Fig. 2). The impact of the counter-anion $\left(\mathrm{SO}_{4}^{2-}\right.$ and $\left(\mathrm{Cl}^{-}\right)_{2}$ were tested) on the $\Delta \lambda$ value was small.

The [L1-Cu-L1] $\mathrm{SO}_{4}$ complex dye (hereafter referred to as reagent $\mathrm{R}$ ) was selected for the subsequent sensing tests performed on the sensitized fibre samples. Studies employing other interesting complexes, especially those containing $\mathrm{Co}^{2+}$, are now in progress and will be published in a separate paper.

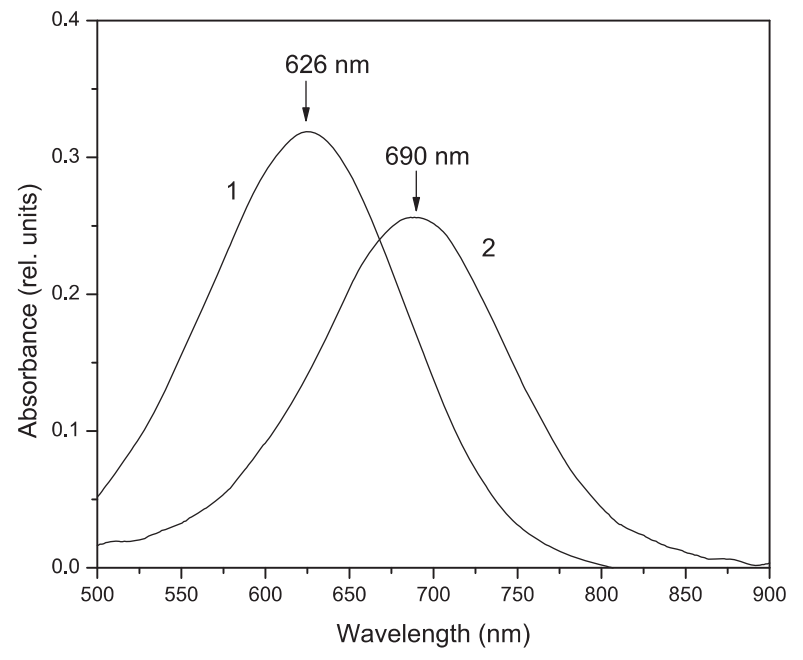

Fig. 1: Absorption spectrum of dye L1 (1) and L2 (2) in ethanol. The solution concentration was $24 \mu \mathrm{M}$ and $19 \mu \mathrm{M}$ for L1 and L2, respectively. The peak positions are indicated.

Table 1: Bathochromic shifts $\Delta \lambda$ resulting from creation of complex ions $[\mathrm{L}-\mathrm{Me}-\mathrm{L}]^{2+}$. The values refer to the absorption maximum of the corresponding ligand $\mathrm{L}(\mathrm{L}=\mathrm{L} 1$ or $\mathrm{L} 2)$ in ethanol.

\begin{tabular}{|c|c|c|c|}
\hline Central ion $\left(\mathrm{Me}^{2+}\right)$ & Counter-ion & $\Delta \lambda(\mathrm{nm}), \mathrm{L} 1$ & $\Delta \lambda(\mathrm{nm}), \mathrm{L} 2$ \\
\hline $\mathrm{Fe}^{2+}$ & $\left(\mathrm{Br}^{-}\right)_{2}$ & 96 & 76 \\
\hline $\mathrm{Co}^{2+}$ & $\left(\mathrm{Br}^{-}\right)_{2}$ & 105 & 82 \\
\hline $\mathrm{Ni}^{2+}$ & $\left(\mathrm{Cl}^{-}\right)_{2}$ & 108 & 70 \\
\hline $\mathrm{Cu}^{2+}$ & $\left(\mathrm{Cl}^{-}\right)_{2}$ & 125 & 70 \\
\hline $\mathrm{Cu}^{2+}$ & $\mathrm{SO}_{4}^{2-}$ & 129 & 70 \\
\hline $\mathrm{Zn}^{2+}$ & $\mathrm{SO}_{4}^{2-}$ & 81 & 69 \\
\hline $\mathrm{Hg}^{2+}$ & $\left(\mathrm{NO}_{3}^{-}\right)_{2}$ & 106 & 80 \\
\hline $\mathrm{Pb}^{2+}$ & $\left(\mathrm{NO}_{3}^{-}\right)_{2}$ & 98 & 68 \\
\hline
\end{tabular}

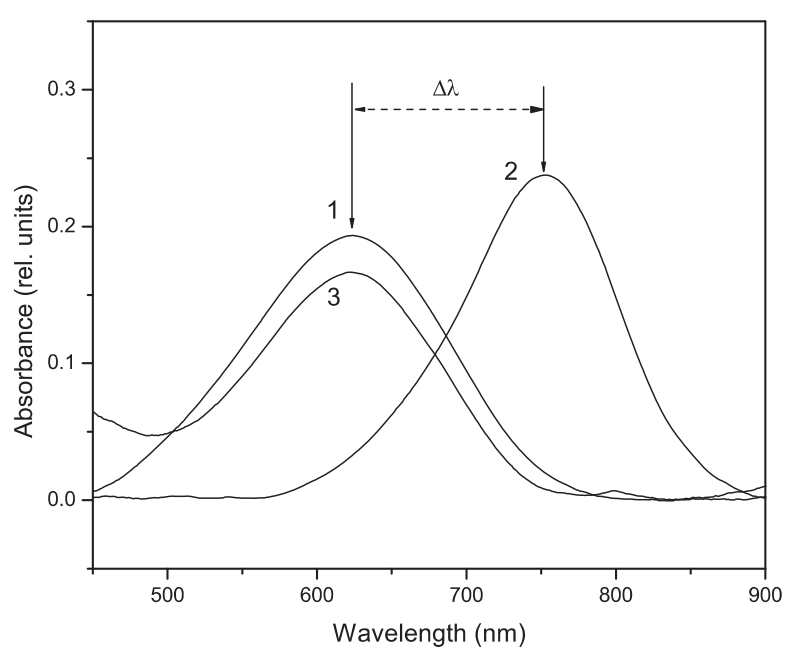

Fig. 2: Absorption spectra of ethanol solutions. Curve 1 - dye L1; 2 - complex reagent $\mathrm{R}=[\mathrm{L} 1-\mathrm{Cu}-\mathrm{L} 1] \mathrm{SO}_{4} ; 3$ - spectrum of $\mathrm{R}$ after addition of ammonia liquor. The bathochromic shift $\Delta \lambda$ accompanying the creation of complexes $\mathrm{R}$ is indicated. 
As expected (cf Eq. 1), the VIS-NIR absorption spectra showed that ion $[\mathrm{L} 1-\mathrm{Cu}-\mathrm{L} 1]^{2+}$ decomposes in contact with ammonia liquor and the ligand absorption spectrum is restored (Fig. 2). A similar reaction was also observed on short fibre sections sensitized with reagent $\mathrm{R}$ and exposed to 10000 ppm ammonia gas (Fig. 3). The forward reaction time $\tau_{90}$ (the time necessary to reach $90 \%$ of the total light intensity drop) was $\sim 30 \mathrm{sec}$, much faster than the fibre recovery time; full spectral relaxation was obtained after $\sim 5$ minutes of nitrogen blow.

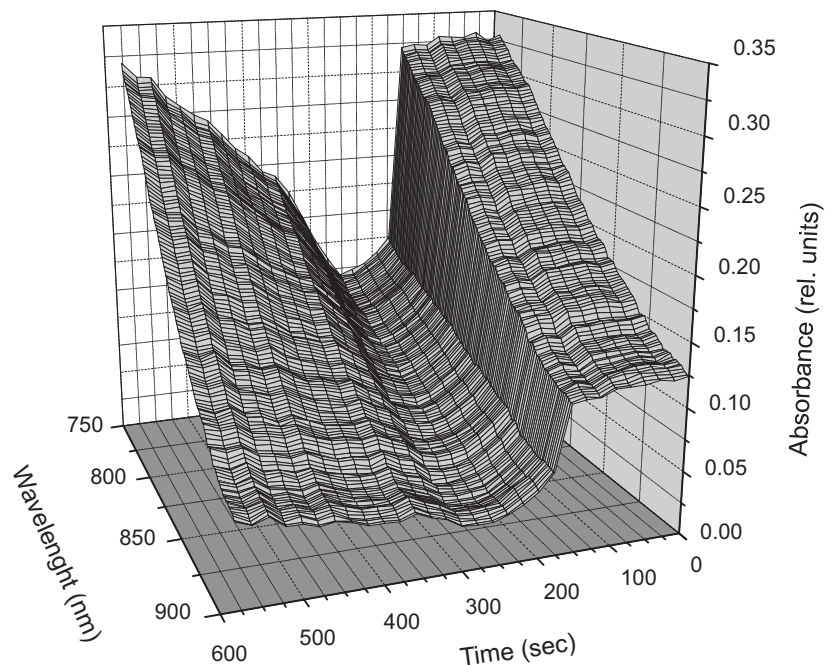

Fig. 3: Absorption spectrum of a short sensing fibre section exposed to dry $\mathrm{NH}_{3} /$ nitrogen gas $(10000 \mathrm{ppm}$, exposure start at $t=160 \mathrm{sec}$ ) followed by nitrogen blow (start at $t=260 \mathrm{sec})$

Ammonia, as a stronger electron donor than the organic ligand, substitutes the latter in the metallic complex, giving rise to a new $\left[\left(\mathrm{NH}_{3}\right)_{\mathrm{n}} \mathrm{Me}\right]^{2+}$ complex ion (cf Eq. 1). The decomposition of the original chromatic complex leads to the observed colour changes. The equilibrium constant of reaction (1) depends not only on the ammonia concentration, but also on the actual degree of dissociation of the individual ionic species. The degree of dissociation varies with the type of ions (metallic ions as well as anions) and with the permittivity of the solvating medium. If a polymer matrix acts as the solvent, the possible diffusion of water into the polymer bulk can also modify the actual degree of dissociation, thus contributing to a remarkable dependence of the sensor signal on the ambient humidity. Such behaviour was indeed experimentally observed $[12,14]$. The presence of hydroxyl and hydronium ions in the polymer matrix can also lead to an alternative chemical process - the creation of an ammonium salt competing with the reaction (1), thus disturbing the sensor function. Careful optimization of the cladding polymer and counter-anion type is therefore necessary to reduce undesired effects. We are currently researching in this direction, and our work will be presented in a forthcoming paper. For this study, only dry gas was used throughout the experiments; thereby reaction scheme (1) could be adopted.

Spectroscopic measurements also confirmed the crucial importance of the initial fibre wash for the long-term spectral stability of the sensitized fibres. The rapid decay of the optical absorption of the reagent $\mathrm{R}$ observed for an unwashed sample (Fig. 4) resulted very likely from a chemical decomposition of the reagent fractions due to a reaction with remains of a UV-initiator/catalyser in the siloxane cladding. The optical properties of fibres properly treated with acetone remained stable for several months of storage in laboratory conditions; the stability and reversibility in field conditions are currently being tested.

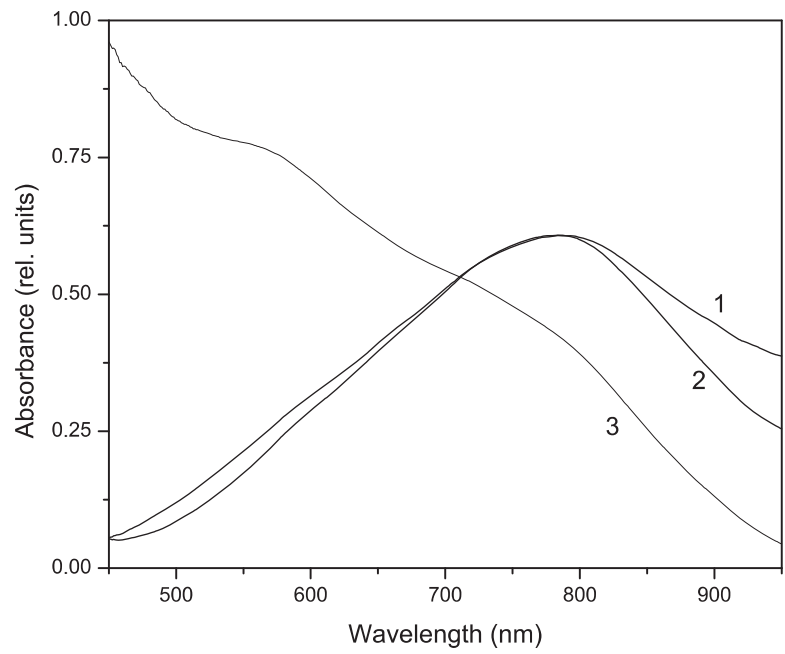

Fig. 4: Absorption spectra of a short sensing fibre demonstrating the influence of initial fibre rinsing in acetone on spectral stability of the reagent after sensitization. Curve $1-$ freshly prepared fibre; curve 2 - after 1 week storage, rinsed fibre; curve 3 - after 1 week storage, non-rinsed fibre.

The optical absorption of a short sensing fibre sample integrated within the spectral interval (840-860) nm decayed with increasing ammonia gas concentration (Fig. 5); the corresponding concentration sensitivity descends with growing analyte concentration and approaches a saturated level at ammonia concentration $\sim 4000 \mathrm{ppm}$. The saturated state likely corresponds to a complete reagent decomposition (cf reaction (1) involving all reagent molecules embedded in the cladding). Thus, increasing the reagent concentration in the fibre cladding could potentially increase the ammonia sensitivity

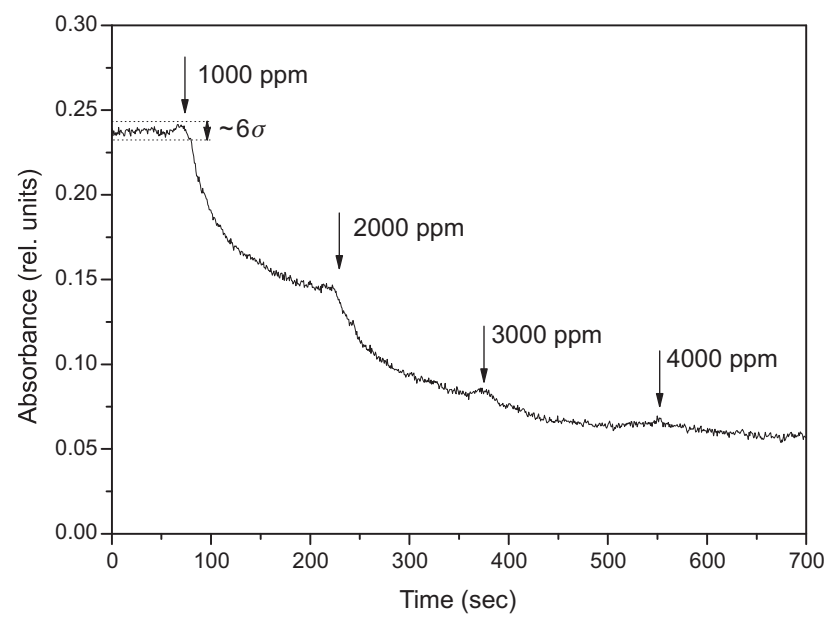

Fig. 5: Absorbance variations measured on a short sensing fibre when exposed to a dry $\mathrm{NH}_{3} /$ nitrogen mixture of increasing concentration. Absorbance values integrated over the spectral interval (840-860) $\mathrm{nm}$. The estimated signal range $6 \sigma$ including $\sim 99.7 \%$ of the signal fluctuations is indicated. 
threshold, but it would also enhance the total fibre attenuation (undesirable for longer fibres), and likely decrease the resolution at low concentrations. This is because the diffusing analyte molecules will mostly react with the reagent molecules located in the outer shell of the cladding, which interacts only weakly with the evanescent field of the fibre core [17]. The cladding thickness and reagent concentration profile therefore also have to be carefully optimized.

The low concentration resolution $r$ within the interval 0-1000 ppm can be roughly estimated from the course in Fig. 5. Assessing the standard deviation (STD) value as $\sigma \cong 0.002$ and taking in account the signal change c. 0.092 we get for $r \cong 1000 \frac{2 \sigma}{0.092} \cong 50 \mathrm{ppm}$. As already mentioned, the practical reagent concentration which can be achieved in a sensing fibre cladding is generally limited. Therefore, the detection resolution can be further improved mainly by reducing the noise level. In our case, we estimate that the primary noise source was the poor light coupling between the tested fibre and the measuring system; the measuring system may be significantly improved if fixing fibre splices are used. Further improvement can also be achieved by applying numeric data accumulation and averaging procedures.

The full OTDR curves recorded with the $120 \mathrm{~m}$ long fibre as fabricated, sensitized by reagent $\mathrm{R}$ within the range (104-110) $\mathrm{m}$, and exposed to $10000 \mathrm{ppm}$ ammonia gas within the same range were dominated by two Fresnel reflections (Fig. 6a). The pulse dispersion along the fibre length is clearly demonstrated by the broadening of the distant (second) reflection. The reflection at $x \sim 0$ comes from the front end of an internal fibre within the Photodyne unit; the second main maximum corresponds to the reflection from the free end of the tested fibre. The tiny side-maximum of the first reflection (at $x \sim 8 \mathrm{~m}$ in Fig. 6a) is caused by the splice connecting the tested fibre to the measuring unit. The fibre sensitization resulted (i) in a slight increase in the intensity back-scattered from the sensitized region (Fig. 6b), followed (ii) by a steeper signal decay from the more distant fibre part combined with a well-resolved reduction in the second Fresnel reflection intensity (Fig. 6, curve 2) compared to the unsensitized state (Fig. 6, curve 1). The first effect may likely be ascribed to the increase in polarizability within the sensitized range enhancing the Rayleigh term $S_{R}(x)$ and reducing $N A(x)$ (cf Eq. $(3,4)$ ). The local drop of the numeric aperture elicits leakage of some part of the guided modes into the cladding, where their scattering and absorption level is much higher than in the fibre core. The second effect results from the enhanced fibre attenuation within the sensitized region ( $\mathrm{cf}$ Eq. 2, 3, 5).

The character of the OTDR signal variation observed after exposure to concentrated ammonia gas (Fig. 6, curve 3) was opposite to the reaction following the sensitization procedure. The second Fresnel reflection grew, the back-scattered signal coming from the sensitized/exposed region decreased, and the slope of the signal that originated just after the exposed region rose. The behaviour is again in accord with the basic OTDR model represented by Eq. $2-5$ and with reaction mechanism Eq. 1. Decomposition of the reagent complexes and the creation of much smaller ions $\left[\left(\mathrm{NH}_{3}\right)_{4} \mathrm{Cu}\right]^{2+}$ led to a local reduction of parameters $\alpha(x)$ and $a^{\prime}(x)$, causing the observed signal changes. The two intersections of curves 2

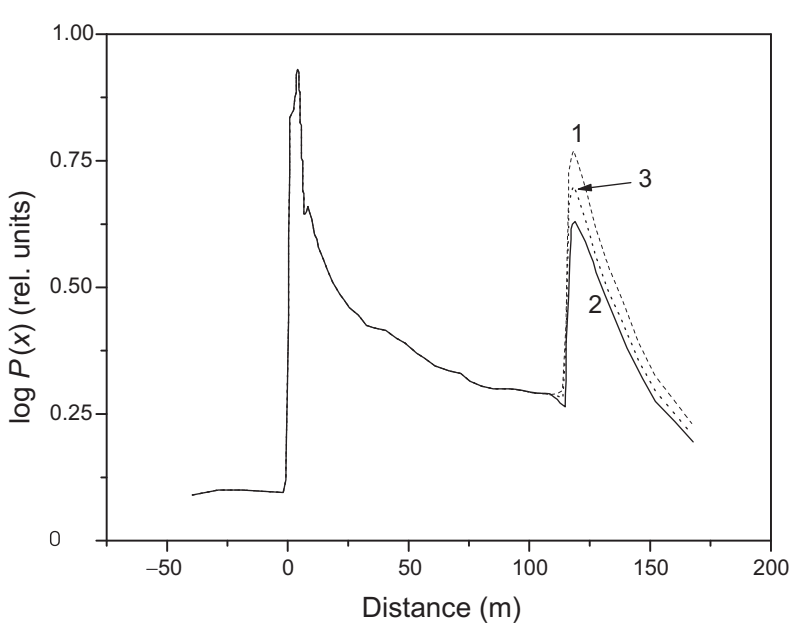

a)

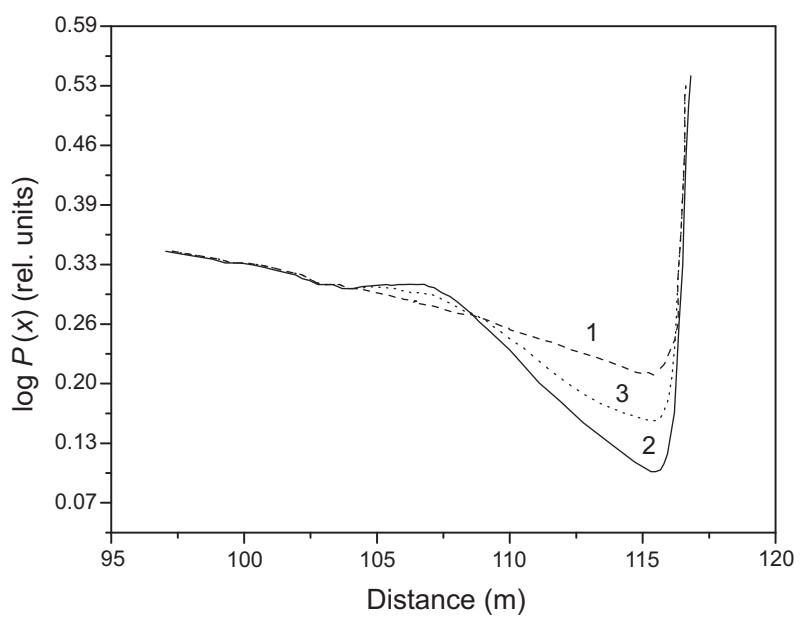

b)

Fig. 6: OTDR curves obtained with a $120 \mathrm{~m}$ long fibre sensitized within distance $x=(104-110) \mathrm{m}$; a) full courses, b) courses corresponding to the sensitized region, recorded with higher resolution (see Experimental section for details). Curve 1 - before sensitization; curve 2 - after sensitization with reagent R; curve 3 - after exposure to 10000 ppm ammonia gas in nitrogen.

and 3 (Fig. 6b) conform very well with the boundaries of the exposed region.

\section{Conclusions}

The results show the principal feasibility of fabricating an ammonia sensing fibre using the selected reagent and fibre sensitization procedure employed here. The sensing parameters obtained with a short fibre sample $\left(r \approx 50 \mathrm{ppm}, \tau_{90} \approx 30 \mathrm{~s}\right)$ are comparable with the figures required for detecting an extensive ammonia gas leakage. We anticipate that comparable sensing parameters would also be achieved with long sensing fibres; the concentration resolution can likely be further improved by using a better optical coupling of the tested fibre to the measuring unit, and numeric signal accumulation and averaging procedures.

The OTDR measurements were performed with the aim to demonstrate the principal feasibility of distributed ammonia gas detection using the proposed fibre design. Two fea- 
tures of the observed OTDR signal are important for the subsequent design of the prototype:

(1) Variation of the Fresnel reflection coming from the free fibre end can be instrumental in detecting any ammonia leak along the fibre length.

(2) The absorbing reagent embedded in the fibre cladding not only enhances the local fibre attenuation (the main factor restricting the maximum length of absorption-based sensing fibres), but also contributes to growth of the local back-scattered light intensity, thus slightly improving the signal-to-noise ratio of the resulting signal.

Our forthcoming research will focus on tests of alternative reagents (such as L1-complex with cobalt) followed by the fabrication of an optimized distributed fibre optic sensor. The static and dynamic characteristics and the long-term stability in field conditions of the sensor will be analysed with reference to the influence of ambient temperature and variations in relative humidity.

\section{Acknowledgment}

Part of this research was supported by the European Commission in the framework of the Copernicus program (contract number CIPA-CT94-0206).

\section{References}

[1] Timmer, B., Olthuis, W., van den Berg, A.: "Ammonia Sensors and their Applications - a Review." Sensors and Actuators. Vol. B107 (2005), p. 666-677.

[2] Zakrzewska, K.: "Mixed Oxides as Gas Sensors." Thin Solid Films. Vol. 391 (2001), p. 229-238.

[3] Shahriary, M. R., Zhou, Q., Siegel Jr., G. H.: "Porous Optical Fibers for High-Sensitivity Ammonia-Vapor Sensors.” Opt. Lett. Vol. 13 (1988), p. 407-409.

[4] Blyler Jr., L. L. et al.: "Optical Fiber Chemical Sensors Utilizing Dye-Doped Silicone Polymer Claddings." Polym. Eng. Sci. Vol. 29 (1989), p. 1215-1222.

[5] Liebermann, R. A.: "Distributed and Multiplexed Chemical Fiber Optic Sensors.” Proc. SPIE. Vol. 1586 (1991), p. 80-91.

[6] Klein, R., Voges, E.: "Integrated-Optic Ammonia Sensor." Sensors and Actuators. Vol. B11 (1993), p. 221-225.

[7] Preininger, C. et al.: "Ammonia Fluorosensors Based on Reversible Lactonization of Polymer-Entrapped Rhodamine Dyes and the Effect of Plasticizers." Anal. Chim. Acta. Vol. 334 (1996), p. 113-123.

[8] Grady, T. et al.: "Optical Sensor for Gaseous Ammonia with Tuneable Sensitivity." Analyst. Vol. 122 (1997), p. 803-806.

[9] Potyrailo, R. A., Hieftje, G. M.: "Optical Time-ofFlight Chemical Detection: Absorption-Modulated Flu- orescence for Spatially Resolved Analyte Mapping in a Bidirectional Distributed Fiber-Optic Sensor." Anal. Chem. Vol. 70 (1998), p. 3407-3412.

[10] Nissilä, S.: "On the Use of Optical Fibres in Industrial sensor Applications." Acta Universitatis Ouluensis C-Technica. Vol. 83, Oulun Yliopisto, Oulu, 1995.

[11] Takada, K.: "Improvement in Signal-To-Noise Ratio of Rayleigh Backscattering Measurement using OLCR." J. Lightwave Tech. Vol. 20 (2002), p. 1001-1017.

[12] Malins, C. et al.: "Fibre Optic Ammonia Sensing Employing Near Infrared Dyes." Sensors and Actuators. Vol. B51 (1998), p. 359-367.

[13] Kvasnik, F et al.: "Rapid Detection and Location of Ammonia Leaks." Final Report on Copernicus Joint Research Project, CIPA-CT94-0206, UMIST, Manchester, 1997.

[14] Scorsone, E. et al.: "Fibre-Optic Evanescent Sensing of Gaseous Ammonia with two Forms of a New Near-Infrared Dye in Comparison to Phenol Red." Sensors and Actuators. Vol. B90 (2003), p. 37-45.

[15] Ingle, J. D., Crouch, S. R.: Spectrochemical Analysis. New Jersey: Prentice Hall, 1988.

[16] Bürck, J., Sensfelder, E., Ache, H.-J.: "Distributed Measurement of Chemicals Using Fiber Optic Evanescent Wave Sensing." Proc. SPIE. Vol. 2836 (1996), p. 1-11.

[17] Saleh, B. E. A., Teich, M. C.: Fundamentals of Photonics. New York: John Wiley \& Sons, 1991.

[18] Klocek, P. et al.: "Measurement System for Attenuation, Numerical Aperture (NA), Dispersion, and Optical Time-Domain Reflectometry (OTDR) in Infrared (IR) Optical Fibers." Proc. SPIE. Vol. 618 (1986), p. 151-158.

Ing. Ladislav Kalvoda, CSc., specialist assistant phone:+420224358606, +420233325508 fax: +420224358601 e-mail: ladislav.kalvoda@fjfi.cvut.cz

Ing. Rudolf Klepáček phone:+420224358 606, +420233 325508 fax: +420224358601 e-mail: rudolf.klepacek@fjfi.cvut.cz

Jan Aubrecht phone:+420 $224358606,+420233325508$ fax: +420224358601

e-mail: jan.aubrecht@centrum.cz

Department of Solid State Physics

Czech Technical University in Prague

Faculty of Nuclear Science and Physical Engineering Trojanova 13

12000 Prague 2, Czech Republic 